(8) The decrease in heat content of transfer of the hydrogen ion from $\mathrm{MeCl}(c)$ in $\mathrm{HCl}(0.1)$ to $\mathrm{HCl}(\mathrm{O} . \mathrm{I})$ is shown to be a function of the total ion activity of the solution, and not of the activity of the hydrogen ion.

Primangr,phta, Pa.

\title{
THE IONIZATION POTENTIAL OF HYDROCHLORIC ACID AND THE ELECTRON AFFINITY OF CHLORINE. ${ }^{1}$
}

Bx Paul D. Foote and F. L. Mohlgre.

Received July $18,1920$.

There are several means conceivable whereby a molecule of hydrogen chloride may be ionized.

r. The structure of the molecule might remain intact, the molecule as a whole losing an electron, as in the case of an atom of monatomic metal. This would require the existence of positively charged molecules of hydrogen chloride. The work of $A_{s t o n}{ }^{2}$ on positive ray analysis indicates that such may be the case. In discharge through phosgene containing hydrogen chloride, he found that ions were present of mass 35 , 36,37 and 38 , and identified these as due to the 2 isotopes of chlorine and their corresponding acids.

2. We have shown ${ }^{3}$ that in the case of hydrogen our experimental data lead to the conclusion that the hydrogen molecule may be ionized by dissociation into atoms and ionization of one atom. If hydrogen chloride were similar to hydrogen in this respect, the ionization might result in a positively charged hydrogen atom and a neutral chlorine atom. The work required to ionize thus a gram molecule of hydrogen chloride may be computed in the following manner.

I et $Q_{\mathrm{HCl}}=$ heat of formation of one gram mol. of $\mathrm{HCl}$ from the diatomic gases $\mathrm{H}_{2}$ and $\mathrm{Cl}_{2}$.

$D_{\mathrm{H}}=1 / 2$ the heat of dissociation of a gram mol. of $\mathrm{H}_{2}$ into monatomic hydrogen.

$D_{\mathrm{Cl}}=1 / 2$ the heat of dissociation of a gram mol. of $\mathrm{Cl}_{2}$ into monatomic chlorine.

Accordingly,

$$
\begin{aligned}
\mathrm{HCl}+Q_{\mathrm{HCl}} & =1 / 2 \mathrm{H}_{2}+1 /{ }_{2} \mathrm{Cl}_{2} \\
1 / 2 \mathrm{H}_{2}+D_{\mathrm{H}} & =\mathrm{H} \\
1 / 2 \mathrm{Cl}_{2}+D_{\mathrm{Cl}} & =\mathrm{Cl}
\end{aligned}
$$

Adding $\quad \mathrm{HCl}+\mathrm{Q}_{\mathrm{HCl}}+D_{\mathrm{H}}+D_{\mathrm{Cl}}=\mathrm{H}+\mathrm{Cl}$.

The quantity $Q_{\mathrm{HCl}}+D_{\mathrm{II}}+D_{\mathrm{Cl}}$ is the work required to dissociate a gram mol of hydrogen chloride into neutral monatomic hydrogen and

1 Published by permission, Director Bureau of Standards.

2Aston, Phil. Mag., 39, 6r. (r920).

${ }^{3}$ Mohler and Foote, J. Opt. Soc. Am, 4, 49 (1920). 
chlorine. To this must be added the work required to ionize a gram atom of hydrogen, $j_{\mathrm{HF}}$. We thus obtain for the work, $/_{\mathrm{HC}}$, necessary to ionize a gram mol. of hydrogen chloride, the following expression,

$$
j_{\mathrm{HCl}}=J_{\mathrm{H}}+()_{\mathrm{HCl}}+D_{\mathrm{H}}+D_{\mathrm{Cl}} \text {. }
$$

All the terms on the right are known. The ionization potential of monatomic hydrogen is 13.5 volts. To express this in kilogram calories per gram atomic weight we use the relation,

whence

$$
\text { kilogram calories }=23.1 \times \text { volts }
$$

$$
\begin{aligned}
& l_{\mathrm{H}}=312 \text { kilogram calories } \\
& Q_{\mathrm{HCl}}=22 \text { (Liddell's Handbook) } \\
& D_{\mathrm{H}}=45 \text { (Langmuir) } \\
& D_{\mathrm{Cl}}=5^{6} \text { (Pier) }
\end{aligned}
$$

Thus, on adding, $/ \mathrm{HCl}=435$ kilogram calories per g. tnol. $\approx 18.8$ volts per molecule.

3. An atom of chlorine is believed to possess an affinity for an electron. That is, a neutral chlorine atom readily unites with an electron so that an amount of energy $E$ must be expended on the negative ion to reduce it to a neutral condition. Until recently, no method was known for determining the magnitude of the quantity $E$. Born, Fajans and others ${ }^{1}$ have lately arrived at a numerical estimate of $E$ through a somewhat complicated and abstract process of reasoning. In order to make our discussion of this work clear, it is necessary to indicate briefly the method pursued by Born.

The compressibility of a crystal of a halogen salt of a monatomic metal may be fairly well explained quantitatively on the assumption that in addition to the ordinary Coulomb force of repulsion or attraction, between the charges on the ions forming the crystal structure, there exists between 2 ions a repulsive force, the potential of which is inversely proportional to the gth power of their distance apart.

It is accordingly possible to compute the potential energy of the crystal system. The negative of this, $U$, referred to a $\mathrm{mol}$ of the crystal, is the work which must be done on a gram molecular weight of the crystal to convert it into free positive and negative ions. Born finds

$$
U=\frac{N a}{4 \delta} \frac{n-\mathrm{I}}{n}
$$

where $a$ is a universal constant, $N=$ Avogadro's number, $n=9$ for alkali salts (except lithium, where $n=5$ ), and $\delta$ is the grating space of the simple cubic crystal, $i$. $e$, the distance between like atoms measured along the edge of an elementary cube. This is readily com-

1 See series of papers in Verh. deut. physik. Ges., Igr8 and $\mathbf{x} 9 \mathbf{9}$. 
puted from the molecular weight, density, and Avogadro number. In this manner we may obtain a table of values of $U$ for a large number of salts. The heats of formation $Q$ of the salts are tabulated in any chemical handbook.

Let $E=$ electron affinity in $\mathrm{kg}$. cal. of the halogen atoms per g. atom.

$J=$ ionization potential in $\mathrm{kg}$. cal. of the neutral metal atoms per g. atom.

$D=$ heat of dissociation per $1 / 2 \mathrm{~g}$. mol. of diatomic halogen gas.

$D^{\prime}=$ latent heat of sublimation of the metal per $\mathrm{g}$, atom.

The work required to dissociate a gram atom of solid metal into free ions and electrons is accordingly $D^{\prime}+J$, while the work required to dissociate $1 / 2 \mathrm{~g}$. mol. of diatomic halogen gas, in an atmosphere of electrons, into negatively charged ions is $D-E$. Hence, the heat of formation, for example, of sodium chloride crystal per g. mol. may be expressed as follows:

$$
Q_{\mathrm{NaCl}}=-D_{\mathrm{Na}}^{\prime}-J_{\mathrm{Na}}-D_{\mathrm{Cl}}+E_{\mathrm{Cl}}+U_{\mathrm{NaCl}}
$$

The values of $J$ for the alkali metals are known from our experimental work ${ }^{1}$ to be accurately determined by the quantum relation $h \nu=e V$, where $\nu$ is the limit of the principal series of each metal in question. Born has shown that the value of $D^{\prime}$ for all the alkali metals is about $20 \mathrm{~kg}$. cal. Hence, the above equation may be solved for $E_{\mathrm{Cl}}$, the only unknown. Proceeding in a similar manner for other chlorides, it is found that the same value of $E_{\mathrm{Cl}}$ is obtained, thus substantiating the theory. The mean value of $E_{\mathrm{Cl}}$ per g. atom was computed as IIg kg. cal., or expressed in volts and referred to a single atom, 5.2 volts.

The second possible method for ionizing hydrogen chloride described above assumed the dissociation into hydrogen ion and neutral chlorine atom. If chlorine possesses an affinity for electrons, the chlorine atom might readily attach an ellectron to itself, since in the process of ionization, 2 electrons are present, the impacting electron and the electron ejected from the hydrogen atom. The ionization of hydrogen chloride would accordingly ultimately result in hydrogen ion and chlorine ion, but the energy disappearing when the chlorine atom becomes negatively charged would not by Method 2 have any effect upon the measured ionization potential of hydrogen chloride. Under such conditions, it should be radiated as a quantum of energy $e E=h \nu$, and the wave length, $\lambda=$ $2400 \AA$ might be detected spectroscopically (if existing).

Born, Fajans, and others are of the opinion, however, that the gain in energy by the production of the negative chlorine atom assists in the ionization of hydrogen chloride. On this basis, the collision of an electron of sufficient velocity with the hydrogen chloride molecule, results in the immediate production of a positively charged atom of hydrogen and a

: Foote, Rognley and Mohler, Phys. Rev., 13, 59 (1919). 
negatively charged atom of chlorine. A mechanical picture of the phenomenon readily suggests itself. The molectle of hydrogen chloride may be represented structurally by $\mathrm{H}^{+}-\mathrm{Cl}^{-}$, and the ionization accordingly consists in a splitting apart of the 2 ions or in a disruption of the bond holding the 2 ions together. The work necessary to accomplish this, deduced in a manner similar to that outlined above, gives the following relation for the ionization potential:

or

$$
J_{\mathrm{HCl}}=J_{\mathrm{H}}+\Omega_{\mathrm{HCl}}+D_{\mathrm{H}}+D_{\mathrm{Cl}}-E_{\mathrm{Cl}}
$$$$
J_{\mathrm{xCl}}=312+22+45+56-\mathrm{II} 9=
$$$$
3 \mathrm{I} 6 \mathrm{~kg} . \mathrm{cal} . / \mathrm{g} . \mathrm{mol} . \mathrm{wt} . \approx 13.7 \text { volts/molecule. }
$$

Born uses slightly different values for some of the above constants and obtains $J_{\mathrm{HCl}}=13.9$ volts, while Fajans computes 14.0 volts. This value is so close to the ionization potential of hydrogen, 13.5 volts, that every precaution must be taken to preclude the production of hydrogen by ordinary chemical reaction in the ionization tube.

\section{Experimental.}

The hydrogen chloride was generated by dropping hydrochloric acid into conc. sulfuric acid. This was passed through calcium chloride and a trap cooled to about $-55^{\circ}$ to eliminate water vapor, and then was allowed to stream through the ionization chamber. A similar trap was mounted between the ionization chamber and condensation pumps in order to reduce the vapor pressure of mercury. In a few cases the experiments were made with the vapor stagnant instead of streaming.

The ionization chamber was of the 4-electrode type, consisting of a hot wire with a cylindrical net surrounding it and at some distance a second concentric cylindrical net and outer cylinder. The electrodes were of platinum and hot cathodes of both platinum and molybdenum were employed since the latter could be operated at considerably higher temperatures and thus furnish a much larger electron current. The inleading wires were of tungsten sealed to Corning glass GT70-702 P-Pyrex, and all metal joints were made either by welding or in some cases, by means of gold. Ordinary silver solder is readily attacked by the vapor.

The general methods of measurement have been discussed in papers on. metallic and non-metallic vapors and gases. ${ }^{1}$

We were unable to show that hydrogen chloride possesses a resonance potential, a property exhibited by all of the other materials so far investigated. Two methods were employed to detect ionization.

${ }^{1}$ Tate and Foote, Phil. Mag., 36, 64 (1918); Bur. Standards Sci. Paper, 317; J. Washington Acad. Sci., 7, 517 (1917); Foote and Mohler, Phil. Mag., 37, 33 (1919); Foote, Rognley, and Mohler, Phys. Rev., r3, 59 (19r9); Mohler, Foote and Stimson, Bur. Standards Sci. Paper, 368; Mohler and Foote, "Non-Metallic Vapors and Gases." Bur. Standards Sci. Paper (in press); J. Opt. Soc. Am., 4, 49 (1920). 
I. The electrons were accelerated between the hot wire and inner net by a potential difference $V$. A portion of these passed through the net into the space between the 2 nets which were maintained at the same potential, and if of sufficient velocity, produced ionization. The positive ions diffused into the space between the outer net and cylinder which were connected through a galvanometer to a potential of such magnitude and sign that no electrons could pass through. The galvanometer accordingly showed zero deflection as the accelerating voltage was increased, until this reached the ionization potential, at which point an ionic current is observed.

2. The second method was that of Davis and Goucher, the details of which have been described in their paper. ${ }^{1}$

In order to correct for the velocity distribution of electrons, voltage drop along the cathode, polarization films, etc., velocity-distribution curves were obtained, using the apparatus as a 3 -electrode tube, the outer net and cylinder being connected together. It is necessary to plot these curves on the proper coordinate scale, such that the galvanometer pos-

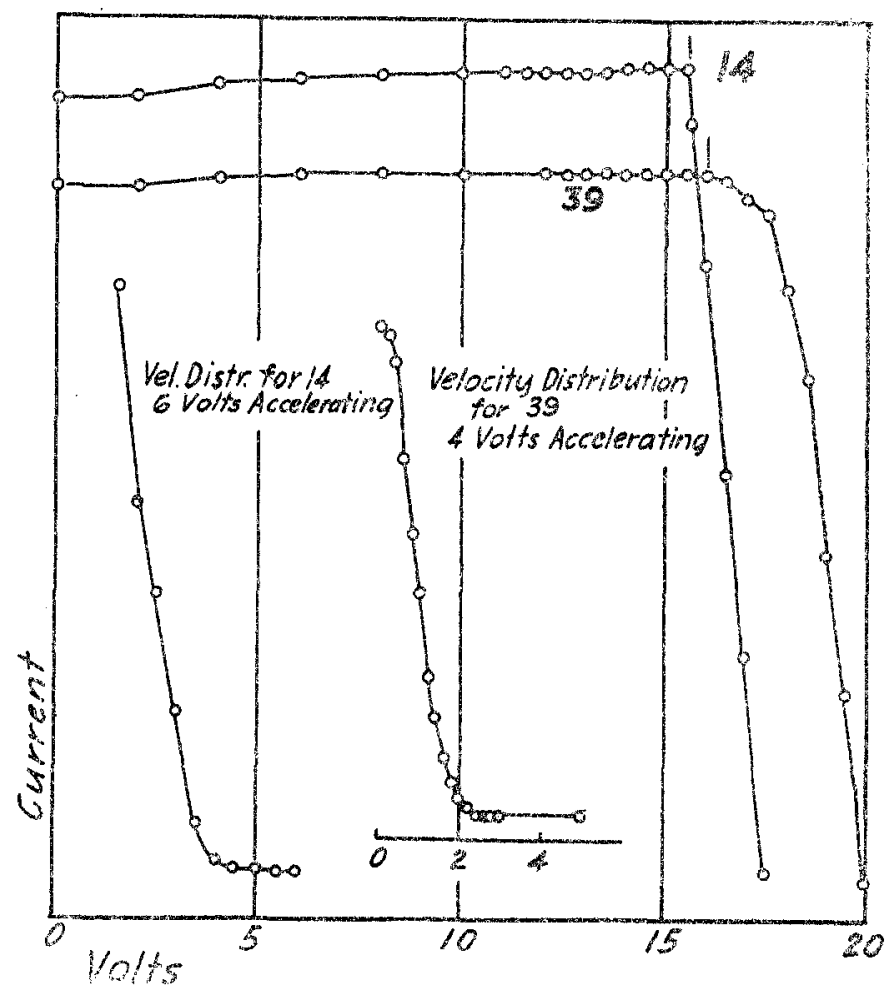

Fig. I-Typical ionization and velocity distribution curves.

1 Davis and Goucher, Phys. Rev., Io, Ior (1917). 
sesses the same sensitivity to ions formed above the ionization potential and to electrons emitted much more copiously at low accelerating voltages with the 3 -electrode tube. Typical curves are illustrated in Fig. I.

Table I summarizes the data for hydrogen chloride, giving observed or applied potentials at ionization, initial potential corrections as determined by the velocity distribution curves, vapor pressure, and corrected values for the ionization potential. The final mean is 14.0 volts with a probable error of 0.2 volt. If we omit all data obtained on humid days when the electrical leaks were troublesome and use only those determined from curves I0, I4, I $8,21,29,34,36,38$ and 40 , the mean value is still 14.0 volts with a probable error of 0. I volt.

TABLE I.

\begin{tabular}{|c|c|c|c|c|c|}
\hline \multicolumn{6}{|c|}{ Ionization Potential of Hydrogen Chloride. } \\
\hline Curve. & $\begin{array}{l}\text { Pressure. } \\
\text { Mm. }\end{array}$ & $\begin{array}{l}\text { Applied potential } \\
\text { at ionization. }\end{array}$ & $\begin{array}{c}\text { Initial } \\
\text { potential. }\end{array}$ & $\begin{array}{l}\text { Ionization } \\
\text { potential. }\end{array}$ & Method. \\
\hline 2 & 0.15 & 13.0 & -0.5 & $\mathrm{I} 2.5$ & I \\
\hline 5 & 0.15 & 14.0 & -0.7 & $13 \cdot 3$ & $\mathbf{r}$ \\
\hline 10 & 0.33 & 16.0 & -1.5 & 14.5 & I \\
\hline 14 & 0.33 & 55.6 & -1.5 & $x_{4} .1$ & $\mathrm{I}$ \\
\hline 18 & 0.45 & $x 6.0$ & -1.5 & 14.5 & I \\
\hline $2 \mathrm{I}$ & 1.60 & $x_{5} .0$ & -1.5 & 13.5 & $\mathbf{I}$ \\
\hline 25 & 0.20 & 15.6 & $-\mathbf{X} \cdot \mathrm{I}$ & 14.5 & 2 \\
\hline 28 & 0.20 & 16.0 & -I.I & 14.9 & 2 \\
\hline 29 & 0.25 & 14.0 & -0.7 & $13 \cdot 3$ & $\mathrm{r}$ \\
\hline 30 & 0.25 & 16.5 & -1.0 & 15.5 & 2 \\
\hline $3 \mathbf{I}$ & 0.25 & 14.0 & $\rightarrow 0.6$ & 13.4 & 2 \\
\hline 34 & 0.50 & 16.0 & -2.0 & 14.0 & I \\
\hline 36 & 0.40 & 15.2 & -1.6 & 13.6 & I \\
\hline 38 & 0.30 & I 5.0 & -1.2 & 13.8 & $\mathrm{r}$ \\
\hline 39 & 0.30 & 16.0 & $-\mathrm{I} .6$ & I 4.4. & I \\
\hline & & M & $\ldots$ & $14.0^{\circ}$ & \\
\hline
\end{tabular}

As this value is close to that determined by us for atomic hydrogen, it is necessary to show that the ionization potential of hydrogen chloride was actually observed. If a liquid-air trap be connected to the ionization tube, thus condensing the hydrogen chloride, the characteristic ionization disappears, showing that the effect is not due to hydrogen produced by ordinary chemical reaction. This is illustrated in Fig. 2. A pressure of hydrogen chloride equivalent to $1.6 \mathrm{~mm}$. of mercury was admitted to the ionization chamber and the apparatus sealed. With the trap maintained at $-50^{\circ}$, Curve 21 was obtained. After some time the trap was cooled its liquid air and Curve 24 , plotted to the same scale of sensitivity was secured. The pressure as shown by the manometer was $0.007 \mathrm{~mm}$., about the order of magnitude of the vapor pressure of hydrogen chloride at this temperature. Only a trace of ionization is present, which may be accounted for by this small pressure of hydrogen chloride.

A further proof that the ionization was not due to hydrogen, which is 
produced to some extent from dissociation by the hot cathode, is the fact that no visible arc could be detected, even with ionization currents of a magnitude at which hydrogen shows a fairly brilliant discharge.

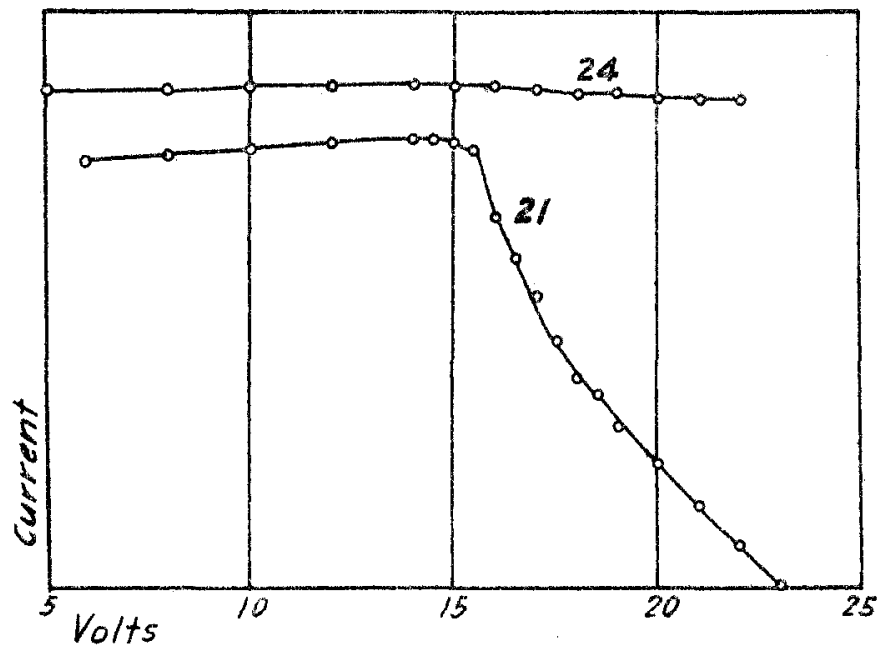

Fig. 2-Curve $2 x$ obtained without liquid air; Curve 24 with liquid air.

A still further confirmation that the inelastic collisions taking place between the 2 nets did not occur with hydrogen is the fact that by the Davis and Goucher method no radiation could be detected below 14.0 volts, while at this voltage and at high pressure, thus permitting a rapid rate of recombination between hydrogen ion and chlorine ion, the radiation, measured photo-electrically, was pronounced. If many, collisions occurred with hydrogen, radiation would have appeared at ro. I volts, the resonance potential of monatomic hydrogen.

We are, therefore, led to the conclusion that but one type of inelastic impact of electrons and molecules occurs in hydrogen chloride. This results in ionization, and the velocity of the impacting electron must be that developed in falling through a potential difference of 14.0 volts.

\section{Conclusions.}

The experimental results indicate that ionization of hydrogen chloride takes place according to Method 3, outlined above. The hydrogen chloride molecule consists of a positively charged hydrogen atom and a negatively charged chlorine atom bonded together, and the ionization simply disrupts this bond. The work necessary to ionize a gram mol of hydrogen chloride is accordingly the work of ionizing a gram atom of hydrogen plus the heat of formation of hydrogen chloride plus the heat of dissociation of 0.5 gram mol. of hydrogen plus the heat of dissociation of 0.5 gram mol of chlorine minus the electron affinity of a gram 
atom of chlorine. This, expressed in volts and referred to a single molecule is 13.7 volts, in excellent agreement with the experimentally determined value of 14.0 volts.

Our experiments accordingly afford a surprising confirmation of the theories of Born, Fajans and others, showing that their deductions on the magnitude of the electron affinities of the halogens from the grating energies of their crystalline salts is well founded. Were it not for the electron affinity of chlorine, hydrogen chloride would ionize at about 18.8 volts, far greater than the observed value. Hence, our value of 14.0 volts leads to an experimental determination of the electron affinity of chlorine expressed by the relation $E_{\mathrm{Cl}}=18.8-14.0=4.8$ volts.

Hydrogen chloride is known to possess no characteristic visible or uitra-violet spectrum, in agreement with natural consequence of the above observed peculiar type of ionization and the observed non-existence of a resonance potential. Any radiation emitted which is characteristic of hydrogen chloride would be produced by the union of hydrogen ion and chlorine ion, giving rise to a quantum of $h v=e V$, where $V$ is 14.0 volts. The corresponding wave length in the extreme ultra-violet is $\lambda=880 \AA$, radiation which might be observed, although the experimental difficulties would be considerable.

Since positively charged hydrogen atoms are formed on ionization of hydrogen chloride, we might expect that on recombination a portion of these atoms would attach electrons to themselves instead of chlorine ions, thus giving rise to the spectrum of monatomic hydrogen. Hydrogen atoms might combine to, form molecules so that some molecular hydrogen is likely produced as a result of electronic impact in hydrochloric acid vapor. A high voltage discharge through hydrogen chloride shows the ordinary spectrum of hydrogen.

It is interesting to note that, as predicted by Haber, the attraction between a hydrogen nucleus and a halogen ion is about the same as the attraction between the hydrogen nucleus and the electron in the neutral hydrogen atom.

BUREAU OF STANDARDS, Washington, D. C.

NOTE.

Correction.-In the article entitled "Reactions of $\beta, \beta^{\prime}$-Dichloro-ethyl sulfide," appearing in the Jume, I920, number of THIS JOURNAL, the following corrections should be made: on p. $122 \mathrm{I}$, in the table, the boiling point should read, $222-3^{\circ}$ at $2 I \mathrm{~mm}$., and the melting point given as Ior $^{\circ}$ should read, $\mathrm{I}_{7 \mathrm{I}^{\circ}}$; and on p. $\mathrm{r223}^{2}$, in the table, the boiling points should read, respectively, $\mathrm{r} 73^{\circ}-5^{\circ}$ at $2 I \mathrm{~mm}$., I93-5 at $22 \mathrm{~mm} ., 223^{\circ} 3^{\circ}$ at $2 I \mathrm{~mm}$., and $170^{\circ}$ at $4 \mathrm{~mm}$. These errata should also appear in the Summary.

O. B. HELFRICH 